\title{
Correspondence
}

\section{TIZANIDINE VS. BACLOFEN IN THE TREATMENT OF SPASTICITY IN PATIENTS WITH MULTIPLE SCLE- ROSIS RE: Can. J. Neurol. Sci. 1988; 15:15-19.}

\section{To the Editor:}

Further study of the tolerance data revealed a slight discrepancy from what we reported in the above publication. In that publication we understated the superior tolerance of tizanidine. Although the efficacy of baclofen appeared to be greater than tizanidine, as judged by physicians and physiotherapists, tolerance of tizanidine was actually superior. We erred in stating that tolerance of baclofen was superior. Although the patients themselves could not perceive a difference in drug tolerance between tizanidine and baclofen, both the investigators and physiotherapists ascertained a significant margin which was significantly in favour of tizanidine (see Table).

\begin{tabular}{lcccccc}
\hline Tolerance Assessment & & & & \\
\hline \hline & & & & & $\begin{array}{c}\text { Comparison } \\
\text { Between } \\
\text { Groups* }\end{array}$ \\
\hline TIZANIDINE & & & & & \\
Patient & $14(24 \%)$ & $13(22 \%)$ & $21(36 \%)$ & $10(17 \%)$ & N.S. \\
Investigator & $11(19 \%)$ & $15(26 \%)$ & $23(40 \%)$ & $9(16 \%)$ & $\#$ \\
Physiotherapist & $11(19 \%)$ & $21(37 \%)$ & $15(26 \%)$ & $10(18 \%)$ & $\#$ \\
BACLOFEN & & & & & \\
Patient & $22(35 \%)$ & $10(16 \%)$ & $19(31 \%)$ & $11(18 \%)$ & N.S. \\
Investigator & $23(38 \%)$ & $6(10 \%)$ & $28(46 \%)$ & $4(6 \%)$ & $\#$ \\
Physiotherapist & $22(36 \%)$ & $10(16 \%)$ & $16(26 \%)$ & $13(21 \%)$ & $\#$ \\
\hline
\end{tabular}

+ Data are expressed as number of patients and $\%$ of total population.

* N.S. = not significant

\# $\mathrm{p}<0.05$

** The numbers include those who dropped out of the study because of adverse reactions (tizanidine 4 ; baclofen 11 )

This doesn't change the essential statement of our study. Both drugs are useful adjuncts in the treatment of spasticity in patients with multiple sclerosis, with slightly different side effects.

G.P.A. Rice

Department of Clinical Neurological Sciences University Hospital

London, Ontario

\section{EFFECT OF BILE DUCT LIGATION-INDUCED LIVER DAMAGE ON THE BLOOD-BRAIN BARRIER}

\section{To the Editor:}

The homeostasis of the CNS is to a great extent controlled by the blood-brain barrier (BBB). Since BBB integrity is maintained by astrocytes, ${ }^{1}$ and since astrocytes are abnormal in hepatic encephalopathy, ${ }^{2}$ one would predict that the BBB would aiso be abnormal in this disease. Evidence from the literature shows BBB dysfunction certainly occurs in acute, severe hepatic encephalopathy. Whether it also occurs in chronic disease, is uncertain, since the evidence is contradictory. The biliary obstruction model of liver damage in the rat represents a disease that is intermediate between acute and chronic. ${ }^{3}$ Biliary obstruction was induced in male Wistar rats by ligating the common bile duct using the surgical procedure described by Franco et al. ${ }^{3}$ Control rats were sham-operated using the same procedure but without ligation and cutting of the bile duct.

Two to three weeks following surgery the animals were anesthetized, the thoracic cavity was opened and $100 \mathrm{mg} / \mathrm{kg}$ of horseradish peroxidase (HRP) (Type Il, Sigma Chemica! Company, U.S.A.), dissolved in $0.9 \%$ saline solution ( 100 $\mathrm{mg} / \mathrm{ml}$ ) injected into the left ventricle of the heart. The animals were decapitated 30 seconds thereafter. Short-term HRP circulation was used for two reasons; a) to avoid spread of extravasated HRP away from the leakage sites and consequent merging of areas positive for reaction product and $b$ ) to avoid the allergic reaction to HRP that occurs in most rats. The left cardiac ventricle was chosen as the injection site in order to deliver HRP to both cerebral hemispheres and to the cerebellum. A maximum of 45 seconds elapsed between opening the thoracic cavity and decapitation. The brief anoxia produced by this procedure does not cause BBB damage.

Brains were carefully removed from the cranial cavities and bisected sagittally. Care was taken to avoid traction on the blood vessels. The non-specific permeability of the BBB in the parieto-occipital cortex and cerebellum was assessed by a) measuring the peroxidase activity in supernatants from tissue homogenates using a spectrophotometric technique, ${ }^{4}$ and b) measuring the density of leakage sites in thick sections of the contralateral hemisphere reacted for peroxidase activity. ${ }^{5}$ For histological studies, paraffin sections of brain and of liver were stained with phloxine and saffron.

The bile duct-ligated rats weighted only $70 \%$ of the body weight of control and sham-operated rats $(p<0.01)$. The ligated rats were lethargic and jaundiced. Their livers, common bile ducts and spleens were dramatically enlarged and their abdominal cavities frequently contained clear xanthochromic fluid. The livers were hard on palpation and of yellow hue. Microscopic examination of the liver tissue revealed that the ligation of the bile duct had resulted in a pronounced proliferation of the intrahepatic bile ducts, single cell necroses and abundant mitoses, accompanied by a chronic inflammatory cell infiltrate and, on occasion, by inconspicuous fibrosis.

No differences in either total HRP activity or leakage site density were found between experimental $(n=20)$ and control groups $(n=19)$, showing that this model of liver disease does not cause defects in the blood-brain barrier. Furthermore, no structural abnormalities were seen in the astrocytes or other tissue elements in the cerebral hemispheres or cerebellar parenchyma from the experimental or sham operated groups. Although our histological examination does not rule out functional abnormalities, the normal appearance of both the BBB and astrocytes in this disease, is consistent with the putative role of astrocytes in barrier maintenance. 
Total peroxidase activity was $16 \%$ higher in the cerebellar tissue than in cerebral cortex $(\mathrm{p}<.005)$ in both experimental and control groups. Measured peroxidase activity includes both intravascular and extravascular compartments. Since tissue samples taken for peroxidase measurements include the rich vasculature of the pia, and since the amount of pia per unit brain tissue is much higher in the cerebellum, it is possible that the higher total peroxidase activity in cerebellum merely represents its larger pial compartment. However, histological measurement of leakage sites did not include leaks that were associated with the pial vasculature, but only intraparenchymatous sites. The number of leakage sites was very low in all samples, in keeping with the normal status of the BBB, but was consistently $50 \%$ higher in cerebellum compared with cerebrum $(\mathrm{p}<<0.001)$. This difference was seen in both experimental and control groups. This apparent leakiness of cerebellar vasculature, compared to cerebral cortex, should be further investigated. It is intriguing to speculate that an increase in BBB leakiness might be associated with the well-known vulnerability of the cerebellum to bloodborne toxins.

\section{J. Neiman \\ L. Resch \\ P.L. Carlen \\ Playfair Neuroscience Institute University of Toronto \\ Toronto, Ontario}

1. Janzer RC, Raff MC. Astrocytes induce blood-brain barrier properties in endothelial cells. Nature 1987; 325: 253-257.

2. Norenberg MD. Hepatic encephalopathy: a disorder of astrocytes. In: Fedoroff S, Vernadakis A, eds. Astrocytes, Vol. 3. Orlando: Academic Press 1986: 425-460.

3. Franco D, Gigou M, Szekley A-M, et al. Portal hypertension after bile duct obstruction. Arch Surg 1979; 114: 1064-1067.

4. Raymond JJ, Robertson DM, Dinsdale HB, et al. Pharmacological modification of blood-brain barrier permeability following in cold lesion. Can J Neurol Sci 1984; 11: 447-451.

5. Stewart PA, Hayakawa EM, Carlen PL. Ethanol and pentobarbital in combination increase blood-brain barrier permeability to horseradish peroxidase. Brain Res 1988; 443: 12-20.

\section{TREMOR IN PATIENTS WITH COMPLICATED MIGRAINE}

To the Editor:

Ischemic events in the territory of the posterior cerebral artery have been implicated in the generation of various movement disorders such as choreoathetosis, tremor, etc. ${ }^{\prime}$ C.M. Fisher has proposed the term "catastrophic migraine" to describe permanent neurological deficits following migrainous attacks. Recently, we have seen three patients who had vascular headaches accompanied by a sudden onset of tremor and in whom an intermittent tremor was the only sequela. One of the case histories is described below.

A 32-year-old woman, with a personal and family history of migraine, came to medical attention because of a tremor of the left upper extremity. Four weeks prior to consulting, she noticed a sudden onset of burning pain in the left shoulder followed by an intermittent tremor of the left arm which occurred twice a week and lasted 1-2 days. Two days after the onset of tremor, she started complaining of severe and tenacious headaches associated with sonophobia and photophobia. On two occasions, the headaches preceded the onset of the tremor by one hour. She also felt that the left side of her body was "like swollen" and this was made worse by headaches and tremor attacks. During the past 2 years, she had one episode of bilateral loss of vision lasting less than 10 seconds. On neurological examination, she had a decreased finger tapping on the left side and a coarse 3-5 $\mathrm{Hz}$ resting tremor of the left arm which was equally present on goal-directed movements and attempts to distract the patient but was slightly reduced in frequency during sleep. On 3 occasions, the tremor completely ceased 30 to 90 minutes following an IV injection of dihydroergotamine. Treatment with nifedipine $10 \mathrm{mg}$ QID was ineffective. However, she had no recurrence of the tremor while treated with both nifedipine $10 \mathrm{mg}$ QID and propranolol $40 \mathrm{mg}$ QID.

Extensive laboratory tests including $\mathrm{CBC}$, sedimentation rate, RA test, ANA, serum complement level, cryoglobulinemia, serum copper and ceruloplasmin, thyroid function tests, ECG, echocardiogram, CSF studies, EEG, and multi-modality evoked potentials were unremarkable. Surface EMG recording revealed a 3-5 Hz alternating tremor of the left arm muscles.

Tremor has rarely been described with migrainous headaches. In an article dealing with unusual vascular events in the territory of the posterior cerebral artery, C.M. Fisher' reported 10 patients in whom a sensory, motor, or visual deficit occurred with severe vascular headaches. In 4 of them, a resting tremor of the paretic extremity appeared 1 to 9 months after the initial event. These vascular events seemed to be of migrainous origin since no embolic source could be found. As compared to Fisher's patients, the tremor in our 3 patients was not associated with a severe neurological deficit, its onset was not delayed, and the fluctuating character of the tremor was striking. The tremor could be present for several days at a time and was on a few occasions temporally related to headaches. Two of our patients described a pain sensation in the trembling extremity and one of them complained of a "swollen" feeling on one side of the body. These descriptions suggest sensory symptoms of thalamic origin although the actual involvement of the latter remains unclear.

The cause of this unusual movement disorder is unknown. However, there is some evidence suggesting that it may be due to a vascular process such as seen in migraine. The laboratory investigation eliminated most known disease processes associated with tremor, namely chorea, systemic lupus erythematosus, Wilson's disease, multiple sclerosis, and emboli to the basal ganglia. Atherosclerosis seems highly unlikely in young women aged 23 to 32 , and recurrence of the movement disorder in the same limb argues against an embolic process. A psychogenic origin also seems unlikely since the tremor persisted during sleep and upon distracting the patient. The presence of intermittent sensory and visual symptoms in young women with a personal and family history of vascular headaches suggests that migraine could be a plausible process. Therefore, we postulate that the tremor seen in our patients is most probably caused by migraine and represents a migraine equivalent.

Sylvie Houde

Sherbrooke, Quebec

Martin Veilleux Montreal, Quebec Division of Neurology Montreal General Hospital

1. Fisher CM. Unusual vascular events in the territory of the posterior cerebral artery. Can J Neurol Sci 1986; 13: 1-7. 